\title{
Operational modal analysis with uncertainty quantification for SDDLV-based damage localization
}

\author{
Michael Döhler ${ }^{1, a}$, Luciano Marin ${ }^{1}$, Laurent Mevel $^{1}$ and Dionisio Bernal ${ }^{2}$ \\ ${ }^{1}$ Inria, Campus de Beaulieu, 35042 Rennes, France \\ ${ }^{2}$ Northeastern University, Department of Civil and Environmental Engineering, Center for Digital Signal Processing, \\ 360 Huntington Avenue, Boston, MA 02115, USA
}

\begin{abstract}
The Stochastic Dynamic Damage Locating Vector (SDDLV) approach is a vibration-based damage localization method based on a finite element model of a structure in a reference state and output-only measurements in both reference and damaged states. A stress field is computed for loads in the null space of a surrogate of the change in the transfer matrix at the sensor positions, where the null space is obtained based on the identified modal parameters in both structural states. Then, the damage location is related to positions where the stress is close to zero. The localization results of this generic approach are perturbed by mainly two sources: modal truncation (not all modes of the structure are available) and modal parameter identification errors (estimation is subject to statistical uncertainties). In this paper, we show how damage localization with the SDDLV approach is improved by taking into account the estimation uncertainties of the underlying identified modal parameters.
\end{abstract}

\section{Introduction}

Over the last twenty years, linear system identification methods found a special interest in structural engineering for the identification of vibration modes and mode shapes of structures, as well as for detecting changes in their vibration characteristics from measured signals. Using output-only measurements from structures in the field, Operational Modal Analysis (OMA) has been successfully used as a complementary technique to the traditional Experimental Modal Analysis (EMA) methods in the lab $[1,2]$. Sensors installed on the structures collect data and statistical approaches that originated from stochastic system realization theory for linear systems provide estimates for the modal parameters of interest (natural frequencies, damping ratio and mode shapes). Changes in these modal parameters indicate damage in a structure [3], and damage localization is possible when linking these changes to a finite element model (FEM) of the structure. Damage localization methods that avoid ill-posed FEM updating, for example, test the data for a change in the parameters of a FEM in statistical test [4-6] or interrogate changes in the flexibility of a structure [7-9].

In this paper we consider the Stochastic Dynamic Damage Location Vector (SDDLV) approach [9], which is a damage localization technique using both finite element information and modal parameters from outputonly vibration measurements. From estimates of the modal parameters in both reference and damaged states, the null space of the difference between surrogates of the transfer matrices at the sensor positions for some Laplace variable $s$ in the complex plane is obtained. Then, a vector in this null space is applied as a load to the FEM of the structure

a e-mail: michael.doehler@inria.fr to compute the stress field, where only the FEM of the structure in its reference state is used. Damage is related to this stress field and is potentially located at elements where the stress is close to zero.

The estimated modal parameters from the output-only measurements that are used for damage localization are naturally subject to variance errors [10-12]. Taking into account these uncertainties proves to be important for structural damage diagnosis. Based on these considerations, a statistical extension of the SDDLV method was developed in [13] for the actual decision if an element is damaged. In [14] a more robust version has been developed, where a joint statistical evaluation of the stress computation is carried out at several Laplace domain variables $s$ where the transfer function matrix difference is evaluated.

Taking into account uncertainties not only improves the statistical robustness of the approach, it also aims at decreasing the number of potential false alarms. In fact, noise, model reduction, sparse instrumentation or other limitations may lead to a set of elements with small stress values (and thus potentially damaged) that is too large to help decision making in real applications. Using uncertainty information will help increasing the contrast between both damaged and undamaged elements.

In this paper, we show how damage localization with the SDDLV approach is improved by taking into account the estimation uncertainties of the underlying identified modal parameters. Based on previous works, the foundation of the SDDLV approach and subspacebased modal analysis with uncertainty quantification is explained in Sects. 2 and 3. Then, the robust approach for the statistical evaluation of the damage localization results is outlined in Sect. 4. Finally, we show application 
results on numerical test cases in Sect. 5, and finish with concluding remarks in Sect. 6.

\section{The SDDLV approach}

The SDDLV approach [9] is an output-only damage localization method based on interrogating changes $\delta G(s)$ in the transfer matrix $G(s)$ of a system, performed between two data sets: one from the undamaged (reference) state and another from the damaged state. Vectors in the null space of $\delta G(s)$ are obtained from system identification results using output-only measurements of both states. Then, they are applied as load vectors to a FEM for the computation of a stress field over the structure in order to indicate the damage location, since it has been shown that these loads induce zero stress (or close to zero in practice) in damaged elements. To gain robustness in this localization approach, the stress field is computed for different values of the Laplace variable $s$ of the transfer matrix, and the results are aggregated. This section summarizes the background of the method.

\subsection{Models}

The behavior of a mechanical structure is assumed to be described by a linear time-invariant (LTI) dynamical system

$$
M \ddot{\mathcal{X}}(t)+C \dot{\mathcal{X}}(t)+K \mathcal{X}(t)=v(t)
$$

where $t$ denotes continuous time, $M, C, K \in \mathbb{R}^{d \times d}$ are the mass, damping and stiffness matrices respectively and $\mathcal{X} \in \mathbb{R}^{d}$ collects the displacements of the $d$ degrees of freedom (DOF) of the structure. The external force $v(t)$ is unmeasured and modeled as white noise. Let the system (1) be observed at $r$ coordinates. As $v(t)$ is unmeasured, it can be replaced with a fictive force $e(t) \in \mathbb{R}^{r}$ acting only in the measured coordinates that re-produce the measured output. With the substitution $x=\left[\begin{array}{ll}\mathcal{X}^{T} & \dot{\mathcal{X}}^{T}\end{array}\right]^{T}$ this leads to the corresponding continuous-time state-space model

$$
\left\{\begin{array}{l}
\dot{x}=A_{c} x+B_{c} e \\
y=C_{c} x+D_{c} e
\end{array},\right.
$$

with the state vector $x \in \mathbb{R}^{n}$, the output vector $y \in \mathbb{R}^{r}$, the state transition matrix $A_{c} \in \mathbb{R}^{n \times n}$ and the output mapping matrix $C_{c} \in \mathbb{R}^{r \times n}$, where $n$ is the system order and $r$ is the number of outputs. The system matrices $A_{c}$ and $C_{c}$ can be obtained from output-only system identification (see Sect. 3). The input influence matrix and direct transmission matrix are $B_{c} \in \mathbb{R}^{n \times r}$ and $D_{c} \in \mathbb{R}^{r \times r}$, respectively, which are not obtained from system identification, but which are relevant for the following developments.

\subsection{Stress computation}

First, vectors in the null space of $\delta G(s)$ are computed using output-only measurements of the structure. The transfer matrix $G(s) \in \mathbb{C}^{r \times r}$ can be expressed without the input influence matrix $B_{c}$ for $2 r \geq n$ as $G(s)=R(s) D_{c}$ [9] with

$$
R(s)=C_{c}\left(s I-A_{c}\right)^{-1}\left[\begin{array}{c}
C_{c} A_{c} \\
C_{c}
\end{array}\right]\left[\begin{array}{l}
I \\
0
\end{array}\right] .
$$

Let the variables in (3) be given in the damaged (variables with tilde) and reference states. The difference in the transfer matrices between both states is $\delta G(s)=\tilde{G}(s)-G(s)$. Then, assuming $\delta D_{c}=\tilde{D}_{c}-D_{c}=0$ (corresponding to no mass change) and $D_{c}$ being invertible, the matrices $\delta G(s)$ and $\delta R(s)^{T}=\tilde{R}(s)^{T}-R(s)^{T}$ have the same null space [9]. Thus, the desired load vector can be equivalently found in the null space of $\delta R(s)^{T}$, which can be obtained from the Singular Value Decomposition (SVD)

$$
\delta R(s)^{T}=U \Sigma V^{H}=\left[\begin{array}{ll}
U_{1} & U_{2}
\end{array}\right]\left[\begin{array}{cc}
\Sigma_{1} & 0 \\
0 & \Sigma_{2}
\end{array}\right]\left[\begin{array}{ll}
V_{1} & V_{2}
\end{array}\right]^{H},
$$

where $U, \Sigma, V \in \mathbb{C}^{r \times r}, \quad \Sigma_{2} \approx 0$ and ${ }^{H}$ denotes the conjugate transpose. The desired load vector $v(s)$ in the null space of $\delta R(s)^{T}$ can be chosen as any linear combination of the vectors in $V_{2}$, in particular as the vector corresponding to the smallest singular value. Note that only output-only measurement data is necessary for the computation of an estimate of $v(s)$.

The computation of the stress (or stress resultants) from the dynamic load vector $v(s)$ requires knowledge of the finite element model (FEM) of the structure. The stress vector $S(s) \in \mathbb{C}^{e}$ at the $e$ elements of the FEM and the load $v(s)$ have a linear relation

$$
S(s)=\mathcal{L}_{\text {model }}(s) v(s)
$$

where $\mathcal{L}_{\text {model }}(s) \in \mathbb{C}^{e \times r}$ is entirely obtained from the FEM of the structure $[13,14]$.

Theoretically, the components of the stress vector $S(s)$ corresponding to a damaged element are zero $[7,9]$ and hence entries in $S(s)$ close to zero indicate potentially (but not necessarily) damaged elements. In practice these stresses are not exactly zero but small due to modal truncation, model errors and uncertainties from the measurement data. Furthermore, since damaged elements lead to stress values that are (close to) zero, but zero stress does not necessarily indicate damage, it is recommended to compute the load vector $v(s)$ and subsequently the stress vector $S(s)$ for several values of the Laplace variable $s$ and to aggregate the results $[7,9]$. Then, the information of the components of the stress vector $S\left(s_{i}\right)$ corresponding to an element $j$ for all $i=1, \ldots, \kappa$ can be used now to obtain the aggregated stress value

$$
\bar{S}_{j}=\sum_{i=1}^{\kappa}\left|S_{j}\left(s_{i}\right)\right|
$$

for each finite element $j$ [9].

Note that while the load vector $v(s)$ is only defined at the $r$ sensor coordinates, damage can be localized at any element of the structure because the stress vector $S(s)$ generated from $v(s)$ covers the full domain. 


\section{Stochastic subspace identification and uncertainties}

\subsection{System identification}

Estimates of the system matrices $A_{c}$ and $C_{c}$ from outputonly measurements of the continuous-time system (2) are required for the damage localization strategy from the previous section. Stochastic subspace-based system identification methods are efficient tools for this task, identifying the discrete-time state-space model

$$
\left\{\begin{array}{c}
x_{k+1}=A_{d} x_{k}+v_{k} \\
y_{k}=C_{d} x_{k}+w_{k}
\end{array}\right.
$$

where $A_{d}=\exp \left(A_{c} \tau\right), C_{d}=C_{c}, \tau$ is the time step and $v_{k}$ and $w_{k}$ are process and measurement noise, respectively. In the first step, we identify estimates $\widehat{A}_{d}$ and $\widehat{C}_{d}$ of the system matrices and subsequently the modal parameters at different model orders from the measurements using covariance-driven subspace identification $[15,16]$. In the resulting stabilization diagram, at most $r$ mode pairs are selected in the second step, such that the condition $2 r \geq n$ is fulfilled. Finally, the corresponding eigenvalues of the continuous-time system and the mode shapes are used to reconstruct the desired estimates $\widehat{A}_{c}$ and $\widehat{C}_{c}$ of the continuous-time system (2) in the last step.

\subsection{Uncertainties}

For the SDDLV damage localization algorithm, estimates of the system matrices $A_{c}$ and $C_{c}$ are obtained in the reference and damaged states from a finite number of data samples. The identification of these matrices is subject to uncertainties due to the unknown excitation (being modeled as white noise), measurement noise and finite data length. Methods for the uncertainty quantification of the estimates from stochastic subspace identification are given in $[11,12]$. With these methods, the empirical covariance of the measurement data is computed from subsets of the available data and propagated to the modal parameters by an analytical sensitivity analysis, resulting in an automated algorithm. In this way, the uncertainties of the system identification from the data measurements are quantified as

$\Sigma_{A_{c}, C_{c}}=\operatorname{cov}\left(\left[\begin{array}{c}\operatorname{vec}\left(A_{c}\right) \\ \operatorname{vec}\left(C_{c}\right)\end{array}\right]\right), \quad \Sigma_{\tilde{A}_{c}, \tilde{C}_{c}}=\operatorname{cov}\left(\left[\begin{array}{c}\operatorname{vec}\left(\tilde{A}_{c}\right) \\ \operatorname{vec}\left(\tilde{C}_{c}\right)\end{array}\right]\right)$

in both reference and damaged states, where vec denotes column-stacking vectorization.

\section{Statistical damage localization}

Since the stress vector estimate $\widehat{S}\left(s_{i}\right)$ is computed from the estimates $\widehat{A}_{c}$ and $\widehat{C}_{c}$ in both reference and damaged states, the uncertainties from the system identification are propagated to uncertainties in the stress vector. These uncertainties may be crucial in deciding whether an element is potentially damaged, i.e. whether the stress of an element is in fact zero or not, while the computed stress value is just close to zero.
In $[13,14]$ the uncertainty propagation from system identification results to the computed stress vectors $\widehat{S}\left(s_{i}\right)$ at the selected the Laplace variables $s_{i}$ was analyzed. In this section, these results are summarized and the respective statistical test is shown.

\subsection{Preliminaries}

The covariance propagation is based on a sensitivity analysis of functions of asymptotically Gaussian estimates $Y=f(X)$. Then, first-order perturbations (used for notational simplicity) yield $\Delta Y \approx \mathcal{J}_{f} \Delta X$ and finally $\operatorname{cov}(Y) \approx \mathcal{J}_{f} \operatorname{cov}(X) \mathcal{J}_{f}^{T}$, where the sensitivity matrix $\mathcal{J}_{f}$ is obtained analytically.

Since we deal with the uncertainties of complex-valued matrices, we introduce an equivalent real-valued notation by defining

$$
M_{\mathrm{Re}} \stackrel{\text { def }}{=}\left[\begin{array}{ll}
\operatorname{Re}(M) & -\operatorname{Im}(M) \\
\operatorname{Im}(M) & \operatorname{Re}(M)
\end{array}\right], \quad M_{\mathrm{re}} \stackrel{\text { def }}{=}\left[\begin{array}{l}
\operatorname{Re}(M) \\
\operatorname{Im}(M)
\end{array}\right]
$$

for any matrix $M$, allowing the uncertainty computation of real-valued matrices.

\subsection{Covariance computation of stress vector}

Suppose that the operational modal analysis step has been made in reference and damaged states, and the covariance of the respective system identification results are available in (7). Then, these covariances are propagated to the stress vector as follows. Note that a more detailed description of this step is available in $[13,14]$.

In a first step, the sensitivity of $R(s)$ in (3) with respect to the system matrices $A_{c}$ and $C_{c}$ is obtained, yielding

$$
\left(\operatorname{vec}\left(\Delta R(s)^{T}\right)\right)_{\mathrm{re}}=\mathcal{J}_{R(s)}\left[\begin{array}{c}
\operatorname{vec}\left(\Delta A_{c}\right) \\
\operatorname{vec}\left(\Delta C_{c}\right)
\end{array}\right]
$$

In the next step, these perturbations are propagated to the load vector $v(s)$ in the null space of $\delta R(s)^{T}=\tilde{R}(s)^{T}-$ $R(s)^{T}$ and finally to the stress vector $S(s)$, as computed in (5). The propagation to the null space vector $v(s)$ is based on the relation $\Delta v(s)_{\text {re }}=\mathcal{J}_{v(s)}\left(\operatorname{vec}\left(\Delta \delta R(s)^{T}\right)\right)_{\text {re }}$, where $\mathcal{J}_{v(s)}$ is derived in [13, Prop. 4]. Then, from (5) it follows

$$
\Delta S(s)_{\mathrm{re}}=\mathcal{L}_{\text {model }}(s)_{\operatorname{Re}} \mathcal{J}_{v(s)}\left(\operatorname{vec}\left(\Delta \delta R(s)^{T}\right)\right)_{\mathrm{re}},
$$

and together with (9) it follows

$$
\Delta S(s)_{\mathrm{re}}=\mathcal{J}_{S(s)} \mathcal{J}_{\tilde{R}(s)}\left[\begin{array}{c}
\operatorname{vec}\left(\Delta \tilde{A}_{c}\right) \\
\operatorname{vec}\left(\Delta \tilde{C}_{c}\right)
\end{array}\right]-\mathcal{J}_{S(s)} \mathcal{J}_{R(s)}\left[\begin{array}{c}
\operatorname{vec}\left(\Delta A_{c}\right) \\
\operatorname{vec}\left(\Delta C_{c}\right)
\end{array}\right]
$$

where $\mathcal{J}_{S(s)}=\mathcal{L}_{\text {model }}(s)_{\operatorname{Re}} \mathcal{J}_{v(s)}$.

A joint statistical evaluation of the stress vector at all chosen Laplace variables $s_{i}, i=1, \ldots, \kappa$, requires the covariance computation of the stacked (real-valued) stress results

$$
\bar{S} \stackrel{\text { def }}{=}\left[\begin{array}{c}
S\left(s_{1}\right)_{\mathrm{re}} \\
\vdots \\
S\left(s_{\kappa}\right)_{\mathrm{re}}
\end{array}\right] .
$$


Stacking (10) for each $s_{i}$ accordingly leads to

$$
\begin{aligned}
\Delta \bar{S}= & {\left[\begin{array}{c}
\mathcal{J}_{S\left(s_{1}\right)} \mathcal{J}_{\tilde{R}\left(s_{1}\right)} \\
\vdots \\
\mathcal{J}_{S\left(s_{\kappa}\right)} \mathcal{J}_{\tilde{R}\left(s_{\kappa}\right)}
\end{array}\right]\left[\begin{array}{l}
\operatorname{vec}\left(\Delta \tilde{A}_{c}\right) \\
\operatorname{vec}\left(\Delta \tilde{C}_{c}\right)
\end{array}\right] } \\
& -\left[\begin{array}{c}
\mathcal{J}_{S\left(s_{1}\right)} \mathcal{J}_{R\left(s_{1}\right)} \\
\vdots \\
\mathcal{J}_{S\left(s_{\kappa}\right)} \mathcal{J}_{R\left(s_{\kappa}\right)}
\end{array}\right]\left[\begin{array}{l}
\operatorname{vec}\left(\Delta A_{c}\right) \\
\operatorname{vec}\left(\Delta C_{c}\right)
\end{array}\right],
\end{aligned}
$$

Since the system matrices from the damaged and reference states are obtained on different statistically independent measurements, the joint covariance of the computed stresses follows as

$$
\begin{aligned}
\Sigma_{\bar{S}}=\operatorname{cov}(\bar{S})=\left[\begin{array}{c}
\mathcal{J}_{S\left(s_{1}\right)} \mathcal{J}_{\tilde{R}\left(s_{1}\right)} \\
\vdots \\
\mathcal{J}_{S\left(s_{\kappa}\right)} \mathcal{J}_{\tilde{R}\left(s_{\kappa}\right)}
\end{array}\right] \Sigma_{\tilde{A}_{c}, \tilde{C}_{c}}\left[\begin{array}{c}
\mathcal{J}_{S\left(s_{1}\right)} \mathcal{J}_{\tilde{R}\left(s_{1}\right)} \\
\vdots \\
\mathcal{J}_{S\left(s_{\kappa}\right)} \mathcal{J}_{\tilde{R}\left(s_{\kappa}\right)}
\end{array}\right]^{T} \\
+\left[\begin{array}{c}
\mathcal{J}_{S\left(s_{1}\right)} \mathcal{J}_{R\left(s_{1}\right)} \\
\vdots \\
\mathcal{J}_{S\left(s_{\kappa}\right)} \mathcal{J}_{R\left(s_{\kappa}\right)}
\end{array}\right] \Sigma_{A_{c}, C_{c}}\left[\begin{array}{c}
\mathcal{J}_{S\left(s_{1}\right)} \mathcal{J}_{R\left(s_{1}\right)} \\
\vdots \\
\mathcal{J}_{S\left(s_{\kappa}\right)} \mathcal{J}_{R\left(s_{\kappa}\right)}
\end{array}\right]^{T}, \quad(12)
\end{aligned}
$$

as detailed in [14].

\subsection{Statistical tests for each element}

The stress vector $\bar{S}$ in (11) contains the computed stress values of all the elements of the structure for all chosen Laplace variables, and the covariance $\Sigma_{\bar{S}}$ in (12) contains its covariance that originates from the uncertainties in the system identification.

Finally, to obtain a meaningful damage index for each structural element $j$, all components of $\bar{S}$ corresponding to element $j$ have to be chosen, as well as the respective rows and columns of $\Sigma_{\bar{S}}$. Let the respective stress vector and (sub-)covariance matrix be denoted as $\bar{S}_{j}$ and $\Sigma_{j}$. Since an estimate of vector $\bar{S}_{j}$ is asymptotically Gaussian distributed [13], it can finally be tested for being 0 and thus potentially damaged with the $\chi^{2}$-test variable

$$
\chi_{j}^{2}=\bar{S}_{j}^{T} \Sigma_{j}^{-1} \bar{S}_{j}
$$

Thus, the scalar variable $\chi_{j}^{2}$ contains a statistically meaningful aggregation of all stress results computed for an element $j$, and it can be decided if an element $j$ is potentially damaged if $\chi_{j}^{2}$ is below a threshold. Such a threshold can be theoretically obtained from the properties of the $\chi^{2}$-distribution for a desired type I or type II error, but for practical purposes it is often sufficient to announce potential damage locations in the elements $j$ with the lowest values of $\chi_{j}^{2}$.

\section{Applications}

The damage localization algorithm with the statistical evaluation of the SDDLV results based on the modal parameter uncertainties has been applied on a simulated plate. All results (aggregated stresses and statistical tests for each element) are normalized to a scale between

\begin{tabular}{|c|c|c|c|c|c|c|c|c|c|}
\hline 91 & 92 & 93 & 94 & 95 & 96 & 97 & 98 & 99 & 100 \\
\hline 81 & 82 & 83 & 84 & 85 & 86 & 87 & 88 & 89 & 90 \\
\hline 71 & 72 & 73 & 74 & 75 & 76 & 77 & 78 & 79 & 80 \\
\hline 61 & 62 & 63 & 64 & 65 & 67 & 68 & 69 & 70 \\
\hline 51 & 52 & 53 & 54 & 55 & 56 & 57 & 58 & 59 & 60 \\
\hline 41 & 42 & 43 & 44 & 45 & 46 & 47 & 48 & 49 & 50 \\
\hline 31 & 32 & 33 & 34 & 35 & 36 & 37 & 38 & 39 & 40 \\
\hline 21 & 22 & 23 & 24 & 25 & 26 & 27 & 28 & 29 & 30 \\
\hline 11 & 12 & 13 & 14 & 15 & 16 & 17 & 18 & 19 & 20 \\
\hline 1 & 2 & 3 & 4 & 5 & 6 & 7 & 8 & 9 & 10 \\
\hline
\end{tabular}

Figure 1. Geometry of the plate with twelve sensor positions and the damaged element.

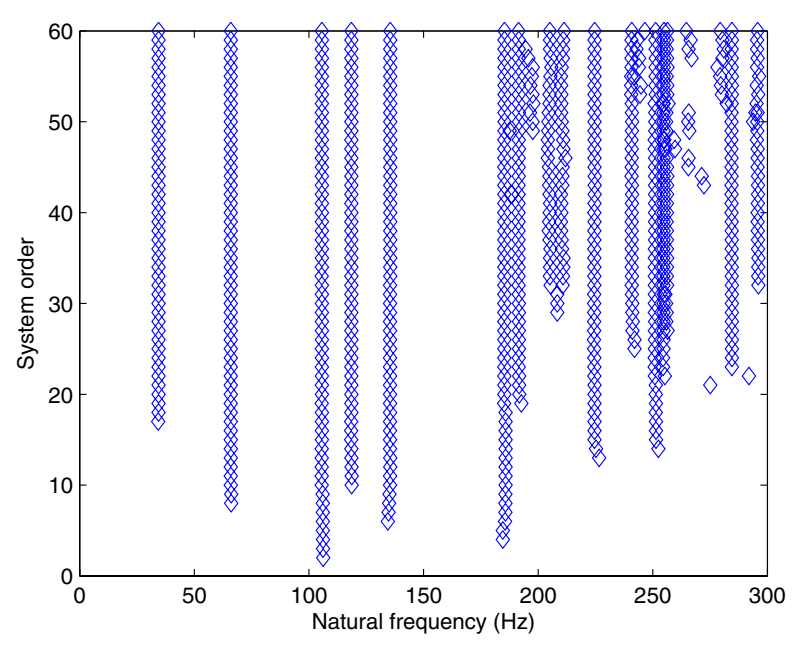

Figure 2. Stabilization diagram from reference state.

0 (possibly damaged, red) and 1 (not damaged, blue) for a visual presentation.

The considered plate model has the dimensions $150 \mathrm{~cm} \times 100 \mathrm{~cm} \times 1 \mathrm{~cm}$. Its edges are fixed and it consists of 100 plate elements as depicted in Fig. 1. Damage in the plate was simulated by decreasing the stiffness in element 66 in several steps by $30 \%$ and $50 \%$ of its original value. For the undamaged and all damaged states, acceleration data of length $N=100,000$ with a sampling frequency of $600 \mathrm{~Hz}$ was generated at twelve sensor positions as shown in Fig. 1. The data was generated from white noise excitation, and $1 \%$ white measurement noise were added on the outputs.

\subsection{Modal analysis and uncertainties}

From the subspace-based system identification (Sect. 3.1) on the simulated data in each structural state, nine well-estimated modes were chosen in the respective stabilization diagrams. The stabilization diagram of the reference state is shown in Fig. 2. 
Table 1. Identified frequencies (in $\mathrm{Hz}$ ) with coefficient of variation (in \%).

\begin{tabular}{r|rrrrrrrrr}
\hline state & 1 & 2 & 3 & 4 & 5 & 6 & 7 & 8 & 9 \\
\hline reference & 34.43 & 65.93 & 105.7 & 118.5 & 135.5 & 185.3 & 191.6 & 224.4 & 284.5 \\
& 0.08 & 0.07 & 0.06 & 0.06 & 0.06 & 0.06 & 0.06 & 0.06 & 0.08 \\
\hline 30\% damage & 34.31 & 65.85 & 105.3 & 118.0 & 135.2 & 185.0 & 191.3 & 224.2 & 283.8 \\
& 0.09 & 0.07 & 0.06 & 0.06 & 0.06 & 0.05 & 0.06 & 0.06 & 0.10 \\
\hline 50\% damage & 34.18 & 65.71 & 105.0 & 117.8 & 135.2 & 184.4 & 191.1 & 224.0 & 283.6 \\
& 0.09 & 0.08 & 0.06 & 0.06 & 0.06 & 0.06 & 0.06 & 0.06 & 0.09 \\
\hline
\end{tabular}

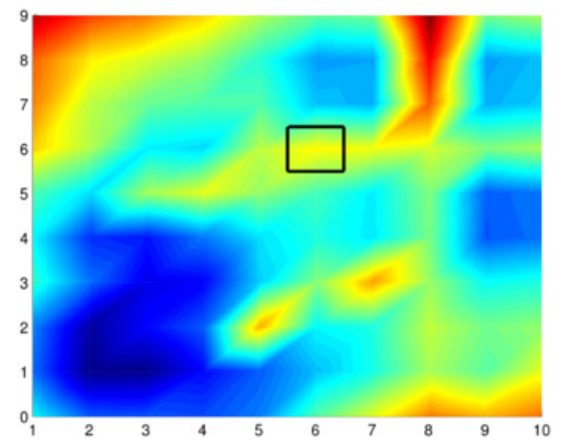

(a) Stress from theoretic modal parameters

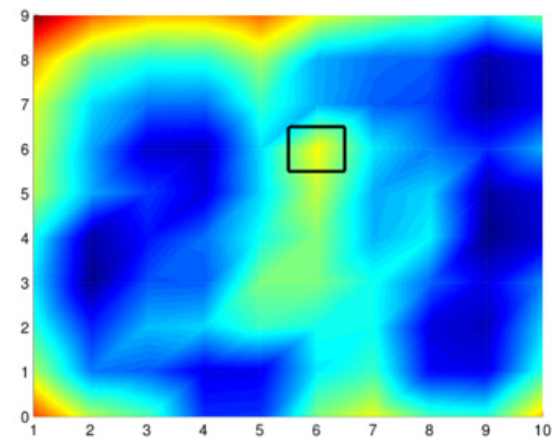

(b) Stress from estimated modal parameters

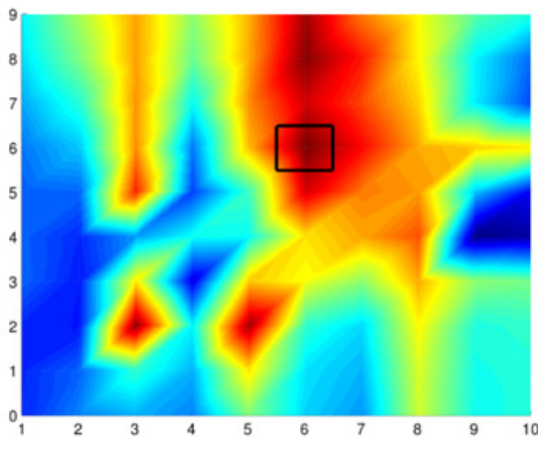

(c) $\chi^{2}$ tests on estimated stress

Figure 3. Stress computation and statistical evaluation at $s=1+140 \mathrm{i}$.

The uncertainties on the estimated modal parameters are obtained as described in Sect. 3.2. The summary of all identified frequencies with their uncertainties is given in Table 1 . Note that the frequency decrease due to damage is less than $0.7 \%$ in the $30 \%$ damage case and less than $0.8 \%$ in the $50 \%$ damage case.

With this information, the matrices $\widehat{A}_{c}$ and $\widehat{C}_{c}$ in the reference and the damaged states are constructed and their covariance in (7) computed.

\subsection{Stress computation}

The matrix $\mathcal{L}_{\text {model }}(s)$ in (5) is obtained from the FEM of the plate model, such that the moments $m_{x x}$ and $m_{y y}$ are computed as the stress resultants for each of the 100 plate elements from the load vector at the sensor coordinates. The different Laplace variables ( $s$-values) considered in these applications are empirically chosen within the range of the identified poles as suggested in [9] and no criterion is available for the pertinence of the choice of such values. We have chosen the values $s_{1}=0, s_{2}=1+40 \mathrm{i}, s_{3}=1+$ $140 \mathrm{i}, s_{4}=1+250 \mathrm{i}, s_{5}=1+400 \mathrm{i}$ and $s_{6}=1+800 \mathrm{i}$. Then, the real and imaginary parts of the stress vector $S\left(s_{i}\right)$ containing these moments and their joint covariance in (12) is computed for these Laplace variables.

\subsection{Theoretic, deterministic and statistical results at one $s$-value}

In a first example, the computation of the stress at one $s$-value only is demonstrated for the $50 \%$ damage case in element 66. To illustrate the difficulties for damage localization that are related to modal truncation (using only nine modes) and estimation errors (uncertainties on modes), the following computations are made:

(a) Exact stress: based on the theoretic modes obtained from the models. Contains only modal truncation (nine modes used).

(b) Stress estimation: based on the estimated modal parameters from the data. Contains modal truncation and estimation errors.

(c) Statistical $\chi^{2}$ tests on stress: based on the estimated modal parameters from the data and their uncertainties. Contains modal truncation, but compensates for estimation errors.

In Fig. 3 these computations are made for $s=1+140$ i. It can be seen that the stress computation from the exact modal parameters in (a) cannot indicate the damage position, due to modal truncation. The same happens for the computation from the estimated modal parameters in (b). However, when taking into account the measurement uncertainties in the statistical evaluation in (c), the damage location is found amongst (several) $\chi^{2}$ values that are close to zero.

In Fig. 4, $s=1+800 \mathrm{i}$ was used for the computation. Note that the damage is now localized perfectly, when the true modal parameters are known in (a). However, the small changes in the estimated parameters due to noise in (b) already lead to errors in the stress computation that prevent the localization. Taking into account the estimation uncertainties in (c) does not improve the situation much.

\subsection{Joint stress evaluation for damage localization}

From the results in the previous section it can be concluded that localization with the SDDLV is sometimes not successful due to modal truncation, even when the 


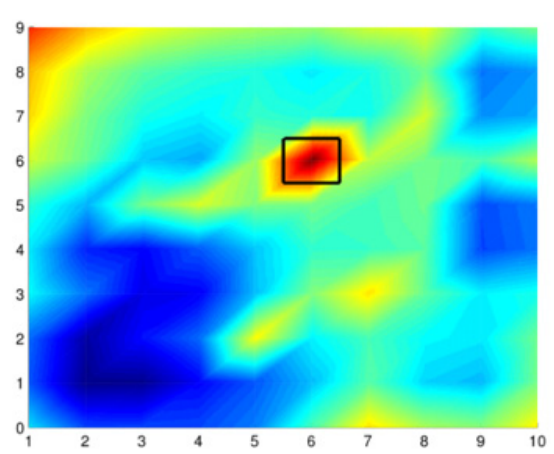

(a) Stress from theoretic modal parameters

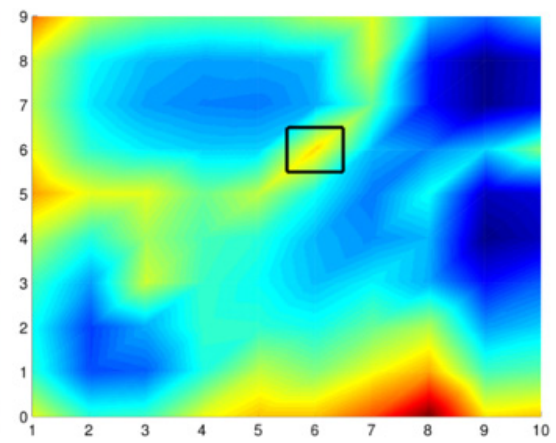

(b) Stress from estimated modal parameters

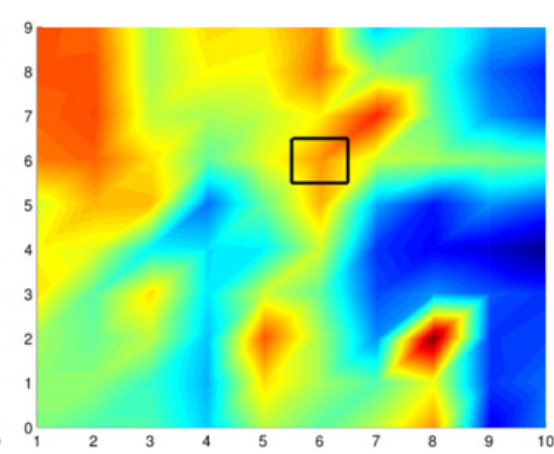

(c) $\chi^{2}$ tests on estimated stress

Figure 4. Stress computation and statistical evaluation at $s=1+800 \mathrm{i}$.

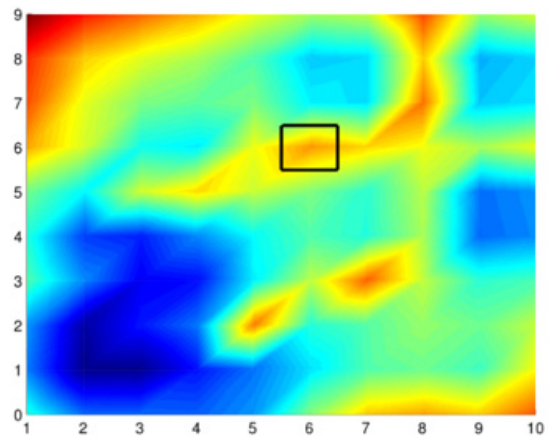

(a) Stress from theoretic modal parameters

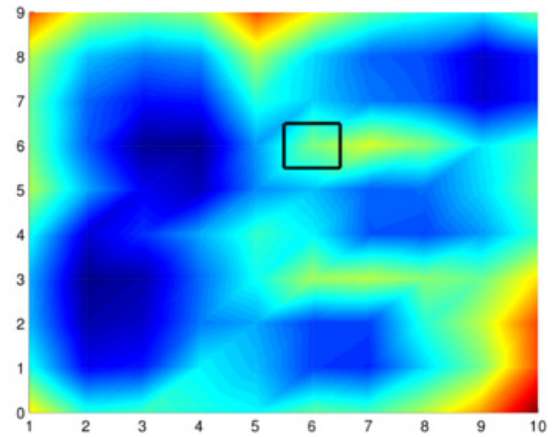

(b) Stress from estimated modal parameters

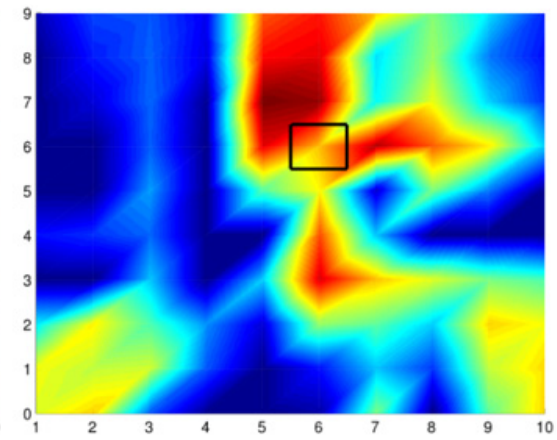

(c) $\chi^{2}$ tests on estimated stress

Figure 5. Joint stress computation and statistical evaluation at six s-values for 30\% damage in element 66 .

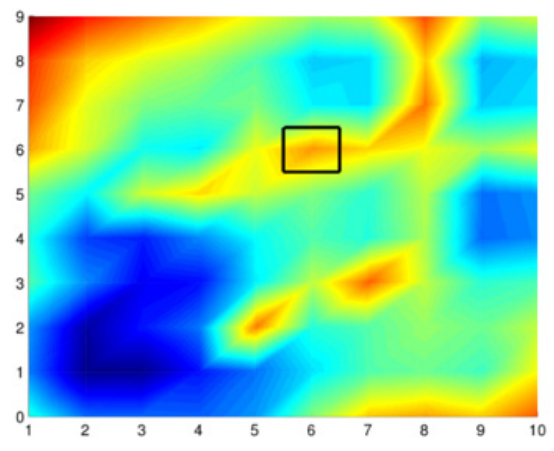

(a) Stress from theoretic modal parameters

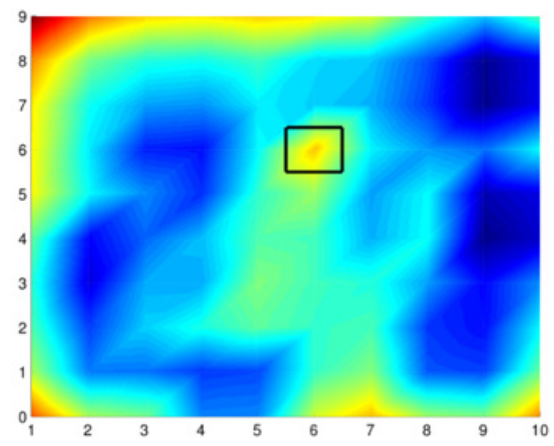

(b) Stress from estimated modal parameters

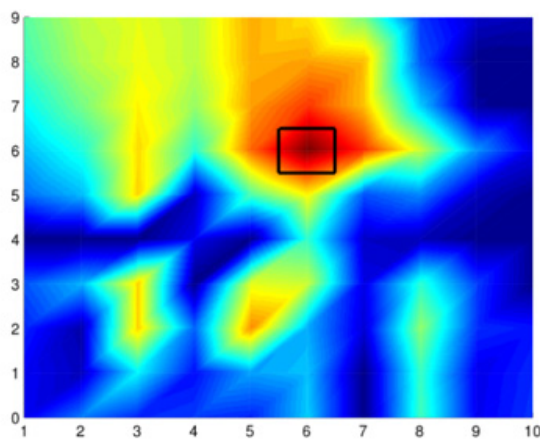

(c) $\chi^{2}$ tests on estimated stress

Figure 6. Joint stress computation and statistical evaluation at six $s$-values for 50\% damage in element 66 .

modal parameters are exact, thus motivating the use of more than one $s$-value for the stress evaluation. Furthermore, considering the statistical uncertainties of the modal parameters for the subsequent stress evaluation can improve the situation significantly, since the variance errors on the estimation are then taken correctly into account and the estimated parameters are weighted in a sensible way.

Hence, we perform a joint stress evaluation at all six $s$-values that are defined above, taking into account the modal parameter uncertainties. In Fig. 5, the results are shown for the $30 \%$ damage case. In this case the damage is found in the neighbouring elements of the damaged element 66 with the statistical evaluation in (c), but not precisely at element 66 due to the low level of damage, while the detected damage is far off without the statistical evaluation even with the exact modal parameters in (a) and with the modal parameter estimates in (b). With 50\% damage in element 66 , the localization is successful with the statistical stress evaluation in Fig. 5 in (c). The damage can be precisely located, while this is not the case without the statistical evaluation.

Note that in all cases the global damage to the simulated structure is very low with less than $0.8 \%$ frequency decrease after damage. With very small damage, the estimate of the surrogate of the transfer matrix difference $\delta R(s)$ deteriorates significantly due to noise, since a substraction of two noisy matrices from reference 
and damaged states is made that are close when the change is small. Still, at this level of damage we were able to localize damage based on the SDDLV method, where the statistical evaluation of the results as well as taking several $s$-values for evaluation proved to be crucial for a precise and robust localization.

\section{Conclusions}

In this paper we have shown how taking into account the uncertainties of estimated modal parameters can improve results of the SDDLV damage localization technique, which requires modal parameters from outputonly measurements in the reference and damaged states of the structure and a FEM in the reference state. While modal truncation and variance errors of the required modal parameters may perturb results, a consistent statistical analysis can at least in part compensate and improve the damage localization resolution. Further details of are found in $[13,14]$.

\section{References}

[1] W. Heylen, S. Lammens, P. Sas, Modal Analysis Theory and Testing (Katholieke Universiteit Leuven, Belgium, 1998)

[2] B. Peeters, G. De Roeck, Stochastic system identification for operational modal analysis: a review, Journal of Dynamic Systems, Measurement, and Control 123, pp. 659-667 (2001)

[3] W. Fan, P. Qiao, Vibration-based damage identification methods: a review and comparative study, Structural Health Monitoring 10, pp. 83-111 (2011)

[4] M. Basseville, L. Mevel, M. Goursat, Statistical model-based damage detection and localization: subspace-based residuals and damage-to-noise sensitivity ratios, Journal of Sound and Vibration 275, pp. 769-794 (2004)

[5] E. Balmès, M. Basseville, L. Mevel, H. Nasser, W. Zhou, Statistical model-based damage localization: a combined subspace-based and substructuring approach, Structural Control and Health Monitoring 15, pp. 857-875 (2008)
[6] M. Döhler, L. Mevel, F. Hille, Efficient computation of minmax tests for fault isolation and their application to structural damage localization, in Proc. 19th IFAC World Congress (Cape Town, South Africa, 2014)

[7] D. Bernal, Load vectors for damage localization, Journal of Engineering Mechanics 128, pp. 7-14 (2002)

[8] E. Reynders, G. De Roeck, A local flexibility method for vibration-based damage localization and quantification, Journal of Sound and Vibration 329, pp. 2367-2383 (2010)

[9] D. Bernal, Load vectors for damage location in systems identified from operational loads, Journal of Engineering Mechanics 136, pp. 31-39 (2010)

[10] M. Raffy, C. Gontier, Statistical asymptotic error on modal parameters in combined deterministicstochastic identification algorithm, Mechanical Systems and Signal Processing 19, pp. 714-735 (2005)

[11] E. Reynders, R. Pintelon, G. De Roeck, Uncertainty bounds on modal parameters obtained from stochastic subspace identification, Mechanical Systems and Signal Processing 22, pp. 948-969 (2008)

[12] M. Döhler, L. Mevel, Efficient multi-order uncertainty computation for stochastic subspace identification, Mechanical Systems and Signal Processing 38, pp. 346-366 (2013)

[13] M. Döhler, L. Marin, D. Bernal, L. Mevel, Statistical decision making for damage localization with stochastic load vectors, Mechanical Systems and Signal Processing 39, pp. 426-440 (2013)

[14] L. Marin, M. Döhler, D. Bernal, L. Mevel, Robust statistical damage localization with stochastic load vectors, Structural Control and Health Monitoring (2014), in press

[15] B. Peeters, G. De Roeck, Reference-based stochastic subspace identification for output-only modal analysis, Mechanical Systems and Signal Processing 13, pp. 855-878 (1999)

[16] M. Döhler, L. Mevel, Fast multi-order computation of system matrices in subspace-based system identification, Control Engineering Practice 20, pp. 882-894 (2012) 